\title{
Challenges in the development and growth of small for gestational age newborns
}

\section{Lorenzo lughetti, Laura Lucaccioni \& Fabrizio Ferrari}

To cite this article: Lorenzo lughetti, Laura Lucaccioni \& Fabrizio Ferrari (2017): Challenges in the development and growth of small for gestational age newborns, Expert Review of Endocrinology \& Metabolism, DOI: 10.1080/17446651.2017.1338137

To link to this article: http://dx.doi.org/10.1080/17446651.2017.1338137

Accepted author version posted online: 02

Jun 2017.

Published online: 12 Jun 2017.

Submit your article to this journal $\pi$

Џll Article views: 1

Q View related articles $\asymp$

View Crossmark data $\asymp$ 


\title{
Challenges in the development and growth of small for gestational age newborns
}

\author{
Lorenzo lughettia, Laura Lucaccioni ${ }^{\mathrm{b}}$ and Fabrizio Ferrari ${ }^{\mathrm{b}}$
}

aPost-graduate School of Paediatrics, Department of Medical and Surgical Sciences of the Mother, Children and Adults, University of Modena and Reggio Emilia, Modena, Italy; ${ }^{b}$ Neonatal Intensive Care Unit, Department of Medical and Surgical Sciences of the Mother, Children and Adults, University of Modena and Reggio Emilia, Modena, Italy

\section{ABSTRACT}

Introduction: Being born with low birth weight (less than $2.500 \mathrm{gr}$ ) is considered a public health problem, with an overall world rate of about $14 \%$. Low birth weight may result from a premature birth (<37 weeks of gestation), from several causes of intra-uterine growth restriction or from a combination of both.

Areas covered: We described how, if weight and/or length at birth are not adequate for gestational age, the newborn is considered 'small for gestational age' and may present several growth, hormonal and developmental peculiarities, possibly due to the growth restriction developed during pregnancy. Expert commentary: We provide a description of the possible mid-term consequences of being born small for gestational age and how to follow and provide care for these babies from a multidisciplinary point of view.
ARTICLE HISTORY

Received 15 March 2017

Accepted 31 May 2017

\section{KEYWORDS}

Birth length; birth weight; development; intra uterine growth restriction; preterm; small for gestational age

\section{Introduction: small for gestational age, former definitions and new frontiers}

The prevalence of low birth weight (LBW) $(<2500 \mathrm{~g})$ newborns raises a public health problem, as it contributes to a variety of short- and long-term adverse effects; including increased morbidity and perinatal mortality, neurodevelopmental disorders, poor growth, and metabolic disorders during adult life.

The World's Children 2003 Report [1] described the overall prevalence of $L B W$ as $14 \%$, with varying rates depending on the region: the highest prevalence was found in South Asia (26\%), while in Europe and in industrialized countries, it is between $7 \%$ and $9 \%$. In 2010, the estimated prevalence of small for gestational age (SGA) births in 138 low-income and middle-income countries was highly variable; the highest prevalence was recorded in south Asia (44.5\%), followed by sub-Saharan Africa (25.5\%) and southeast Asia (24.3\%). The greatest numbers of term-SGA infants were born in south Asia (16.2 million) and sub-Saharan Africa (7.5 million). A major challenge in estimating the global burden of babies born SGA is the selection of a common reference population and, in particular, limitations of available fetal growth references [2].

LBW may result from a condition of intrauterine growth retardation (IUGR-intrauterine growth restriction), of preterm delivery or a combination of both. SGA refers to a newborn infant whose weight and/or length at birth is at least 2 Standard Deviations (SD) below the mean for the infant's gestational age. According to this definition, it is considered as SGA when a neonate has a LBW but normal birth length (BL) or a short in length one with normal BW. Although in many countries there is lack of data on the proper incidence of children born SGA, the worldwide estimated range is from $2 \%$ to over $20 \%$, depending also on the definition used [3].

During the past decades, several different definitions have been used to classify SGA newborns, setting the cutoff at less than the 10th centile, as for the World Health Organization (WHO), or less than the 3rd centile. In 2007, the Consensus Statement of the International Societies of Pediatric Endocrinology and the Growth Hormone Research Society recommended considering SGA newborn with weight and/or length below -2 SD or 3rd percentile, as this definition allows to identify the majority of the individuals at risk of short stature in adulthood and may require appropriate auxological follow-up during childhood and later adolescence [4].

Generally, SGA newborn infants may be classified in two different categories: symmetrical (proportional; 30\%), where weight, length, and head circumference are all reduced; and asymmetrical (70\%), where BW is reduced, but linear growth and head circumference are generally preserved. In either group, measurement of head circumference is also indicated as a measure of brain development. Babies with decreased head circumference may experience neuropsychological problems and lower IQ scores during later development [5-7].

These two different conditions are usually linked to the timing of the intrauterine growth restriction (IUGR) onset. The term IUGR has been and is sometimes still used as a synonym of SGA, but it represents a different clinical condition referring to fetuses that have a deviation of intrauterine growth curve in at least in two consecutive assessments by prenatal ultrasound but that are not necessarily SGA. In fact, while the term SGA has statistical roots including both those who have failed in achieving their growth potential, and those 
who are constitutionally small but healthy, the term IUGR refers to an anthropometric longitudinal evaluation through prenatal serial ultrasounds, and defines fetuses with a failure to achieve their growth potentiality due to prenatal pathological factors. According to these definitions, not each SGA newborn is IUGR and, vice versa, not every IUGR newborn will be SGA [8].

The main causes of IUGR are summarized in Table 1. Apart from infections, that may be considered both maternal and fetal causes and may occur at any time during pregnancy, fetal causes (genetic abnormalities, chromosomal, congenital anomalies) appear as precocious signs of IUGR, leading to reductions of all body sizes (weight, length, and head circumference) of the fetus and newborn (proportionate IUGR newborns, 'symmetrical') and most of the times reduced growth of postnatal recovery (catch-up growth). Placental causes may influence the last stages of pregnancy, inducing more frequently a reduction of the BW, with normal or slightly reduced head circumference and length (disproportionate IUGR infants, 'asymmetrical') and usually a good catch-up growth $[9,10]$.

These underline the important diagnostic role played by the biometric prenatal evaluation performed by ultrasounds, which allows both transversal and longitudinal measurements during pregnancy. Moreover, the use of ultrasound with Doppler also allows to identify abnormalities of fetal-placental and of the utero-placental circulations. Nevertheless, it should be taken into account that it is difficult to estimate gestational age through ultrasonographic evaluation due to the smaller size of the IUGR fetus. The identification of SGA infants and/or IUGR is important, seen their increased morbidity and mortality during the neonatal period and later in life (Table 2).

Small size at birth or in infancy is associated with increased morbidity and mortality from cardiovascular and metabolic diseases in adulthood (coronary artery disease, hypertension, stroke, insulin resistance, glucose intolerance, diabetes mellitus). These observations have introduced the concept of 'intrauterine programming,' where adult diseases are initiated by adverse environmental conditions at critical periods of development, in particular during fetal life.

Table 1. Main causes of intra-uterine growth restriction.

\begin{tabular}{|c|c|}
\hline Maternal & $\begin{array}{l}\text { Maternal age (<16 years; }>35 \text { years)Unfavorable socioeconomic } \\
\quad \text { conditions } \\
\text { Parity } \\
\text { Malnutrition } \\
\text { Substance of abuse (smoking, alcohol, drugs) } \\
\text { Hypertension, preeclampsia } \\
\text { Chronic diseases: diabetes mellitus, endocrine disorders, collagen } \\
\quad \text { diseases, kidney disease, cardiovascular disease, anemia. } \\
\text { Tumors } \\
\text { Malformations } \\
\text { Uterine malformations } \\
\text { Infections (particularly complex TORCH) }\end{array}$ \\
\hline Placental & $\begin{array}{l}\text { Anatomical abnormalities } \\
\text { Insertion anomalies (placenta previa, occult detachment) } \\
\text { Anomalies funiculars (chronic compression, thrombosis of the } \\
\text { umbilical vessels, insertion velamentosa) } \\
\text { Insufficient utero-placental perfusion }\end{array}$ \\
\hline Fetal & $\begin{array}{l}\text { Chromosomal abnormalities } \\
\text { Genetic diseases } \\
\text { Congenital metabolic diseases } \\
\text { Infections (particularly complex TORCH) }\end{array}$ \\
\hline
\end{tabular}

Table 2. Perinatal and long-term complications of the SGA/IUGR newborn

\begin{tabular}{ll}
\hline $\begin{array}{c}\text { Perinatal complications in term } \\
\text { and preterm newborn SGA/ }\end{array}$ & hypoxia, perinatal asphyxia, congenital \\
IUGR & anomalies) \\
Perinatal asphyxia & Meconium aspiration syndrome \\
hypothermia \\
Persistent pulmonary hypertension \\
pulmonary hemorrhage \\
Hypoglycemia \\
Hyperglycemia polycythemia/ \\
hyperviscosity \\
Necrotizing enterocolitis (NEC) \\
Thrombocytopenia \\
Neutropenia \\
Increased number of days of \\
hospitalization at birth \\
Increased frequency of readmission to \\
the hospital \\
Reduced catch-up growth (SGA \\
symmetrical) \\
Minor neurological disorders \\
Increased incidence of attention deficit \\
hyperactivity disorder (ADHD) \\
Greatest risk of severe neurological \\
impairment in preterm infants \\
Endocrine and metabolic disorders \\
(insulin resistance/hyperinsulinemia, \\
dyslipidemia, obesity, diabetes type II) \\
Hypertension \\
Cardiovascular diseases \\
\end{tabular}

Most of the children born SGA recover their growth within the first two years of age, although in few babies it can take up to 4 years to attain a height within the normal range. It is considered that about $8-15 \%$ of SGA, especially the symmetrical, fail to show catch-up growth, resulting in short adult stature in most of the cases [11,12]. Moreover, SGA children may have low lean mass and increased central adiposity associated to a higher risk long-term morbidity related to insulin resistance (IR) and metabolic syndrome (MetS) [13].

Thanks to the advances in perinatology and neonatology over the last decades, survival rates of very low birth weight (BW $<1.500 \mathrm{gr}$ ) (VLBW) and extremely low birth weight (BW $<1000 \mathrm{gr}$ ) (ELBV) premature infants has dramatically increased [14]. Of the 135 million live births worldwide in 2010, WHO estimates that 14.9 million babies were born prematurely, representing an increasing burden with a preterm birth rate of $11.1 \%$ [15]. Preterm birth can be also subdivided on the basis of gestational age (GA), as extremely preterm (EPT) occurring at less than 28 weeks of GA, very preterm (VPT) from 28 but less than 32 weeks, moderate preterm (MPT) occurring from 32 and $33+6$ and late preterm (LPT) from 34 to $36+6$ weeks of gestation [16]. The use of birth weight as a selection criterion has the disadvantage to include, in the same study group, more mature children born SGA and preterm infants born appropriate for GA (AGA), that may present slight differences in terms of clinical outcomes [17]. Moreover, in a period that is usually characterized by rapid intrauterine growth, the premature birth leads the neonate to survive in an extra-uterine environment, shifting energy expenditure from growth-promoting actions to survival strategies, often resulting in postnatal growth restriction also named extra-uterine growth restriction (EUGR). EUGR is considered as growth values $\leq 10$ th percentile of intrauterine growth expectation, 
based on estimated postmenstrual age in premature (2334 weeks' estimated gestational age) neonates at the time they are discharged from the hospital [18]. In a large study from 2008, the prevalence of EUGR at 37-42 weeks of GA in premature infants was inversely proportional to the GA at birth, achieving peaks of $90 \%$ for neonates born at 23-26 weeks [19]. Both SGA and AGA neonates may develop an extra-uterine growth restriction, according to the timing of birth and to the clinical complications. Moreover, the timing of extra-uterine growth restriction of a preterm AGA may occur at the same time as the IUGR of the full-term baby SGA/IUGR. It would therefore be important to evaluate whether these two groups of infants may have a similar metabolic and auxological outcome. Accordingly EUGR children who do not show postnatal catch-up growth in early childhood may be considered as potentially eligible for GH therapy [20].

The use of appropriate anthropometric charts is mandatory although modalities of growth change according to intra- and extra-uterine growth. Optimum growth of preterm infants is considered to be equivalent to intrauterine rates since a superior growth standard has not been defined [21].

Achieving term age for a preterm infant (from 37th to 40th weeks of corrected age), generally means increasing weight and length gain, while in-utero growth trend decreases during the very last weeks of gestation. In fact, the PreM Growth Study revealed that although the pattern of preterm infant growth was generally consistent with intrauterine growth, the biggest deviation in weight gain velocity between the preterm infants and the fetus and infant was just before term, between 37 and 40 weeks. Intra uterine growth charts deriving from fetal measurements obtained by ultrasonography, reflected the expected growth for each gestational age without the effects of prematurity. Their disadvantage is the sensitivity of ultrasonography to assess fetal weight, especially during the first weeks of gestation [22,23]. In 2003, Fenton put together data of three different populations and developed an intrauterine growth chart starting at 22 weeks of gestation with a scale of weight every $100 \mathrm{~g}$. The CDC-2000 growth data between 40th and 50th weeks after conception were added resulting in a fetal-neonatal growth chart, which facilitates the adjustment of growth to prematurity, recommended up to 3 years of age [24]. A new version was released in 2013 with data from six countries. It is now a gender-specific growth chart from 22-week gestation until 10 weeks after term and aligns with the WHO-2006 growth charts allowing a longer period of growth follow-up [22]. However, these growth charts are growth references and are not a growth standard, due to the fact that the population studied was not selected to be healthy. International data on the estimated fetal weight (EFW) and fetal biometry are now available from the first results of the INTERGROWTH-21st study, and will complete the Fetal Growth Standards to be used worldwide. The INTERGROWTH-21 prescriptive approach is in contrast to the descriptive approach adopted in the WHO prenatal growth charts. In fact, Intergrowth-21 developed a new fetal weight estimation model using data from mothers and pregnancies which met minimum criteria in regard to age, height, weight, diet, preexisting medical conditions and after excluding those who developed any antenatal complications who may have affected fetal size. The WHO EFW included growth related antenatal complications and made use of a preexisting weight estimation model to construct references. The clinical suitability of the INTERGROWTH-21 and WHO EFW references in independent populations from different parts of the world has not yet been assessed, and needs further studies. It is also important to bear in mind Gardosi's point of view, that for epidemiological analysis as well as for prospective assessment of fetal growth, individual adjustments of weight limits (the so-called customized assessment) reduce false-positive results and help to identify those babies who are pathologically small. This should lead to improved screening and further investigation (especially by Doppler analysis) of those babies who are at risk $[10,25]$. Italian neonatal anthropometric charts, the so-called INeS (Italian Neonatal Study) charts, based on data from more than 45,000 babies, have been available since 2010 . As for the international data mentioned before, these charts do not refer to a healthy target population: the presence of fetal or maternal pathologies with effects on intrauterine growth was not a criterion for exclusion. New INeS charts, adjusted for gender and parity and based on a healthy population, are mandatory to standardize the auxological measurements of Italian babies and provide reliable background information for follow-up [26,27].

\section{Growth in SGA}

Most children born SGA recover percentiles of weight and height after discharge, although they reach a final adult height that is about 1 SD lower than the target height $(\mathrm{TH})$ calculated from parents' height [12]. The typical accretive pattern is characterized by a period of accelerated linear growth during the first 12 months of life (catch-up growth) that fades around 2 years of age [28]. The recovery is considered completed when they reach their genetic potential determined by the height of the parents. However, 10-15\% of those born SGA will continue to present short stature (height $\leq-2 \mathrm{SD}$ ) during childhood and adult life. Severe preterm infants and those with severe degrees of growth retardation, particularly short length at birth, are less likely to reach a stature within the normal range, although $\mathrm{TH}$ plays an important role. The genetic influence on catch-up growth appears to start from the onset of childhood: being born short or becoming short during the first 2 years of life is similar in terms of risk for adult short stature [29]. The first year of life is considered a very critical period for preterm babies due to the long hospital staying and to all the possible severe clinical complications they may go through (bronchopulmonary dysplasia, necrotizing enterocolitis, intraventricular hemorrhage, sepsis, meningitis). Growth in weight and length may be strongly be influenced by these complications, delaying the achieving of the so called full enteral feeding. Preterm who survive the hospital discharge are shorter and lighter than full-term peers, despite the intense catch-up growth they may have had [30]. Although preterm born SGA may take up to four years to achieve a height in the normal range for population, about $80 \%$ of preterm children show growth recovery during the first 2 years of life and, at 3 years of age, approximately $80 \%$ reaches the normality for head circumference and $70 \%$ for weight [31,32]. Long-term follow-up studies on very and extremely preterm infants, as the 
English UPICURE study, showed that birth weight for GA is still strongly associated with growth outcomes at preschool and school age. They have found, at 6 years of age, that children born extremely preterm were still leaner, shorter and with a smaller head circumference than their peers, with some catchup growth observed from 30 months to 6 years of age [33]. The pathophysiology of postnatal growth failure is complex: different anomalies in the GH-IGF1 axis had been described. For this reason, the GH-IGF1 axis has been largely studied in SGA children. Classic growth hormone deficiency is rare in this population, although differences in diurnal $\mathrm{GH}$ secretion patterns compared to AGA controls have been described: GH peaks of production present lower amplitude and increased frequency. When a stimulation test is performed SGA children present lower peaks of $\mathrm{GH}$ secretion, although within normal range [34,35]. Mean IGF-I and IGF-binding protein-3 levels are reduced in SGA children by approximately 1SD, but the range of levels is wide, indicating possible heterogeneity in the mechanisms of growth failure from insufficient IGF-I generation to IGF-I insensitivity [36]. To the best of our knowledge, SGA populations involved in these studies are not divided into many different categories, according to the reason of intra uterine restriction. In fact, children with genetic or anatomical anomalies are excluded, and that most part of the studies are considering SGA newborns due to placental- and/or infection-mediated etiology of restricted fetal growth.

In infants born prematurely, the period equivalent to the third trimester of gestation occurs extra-utero, with higher risk of alterations of the $\mathrm{GH}$ and insulin-like growth factor system, although related data are still controversial [37]. Recently, medical attention regarding SGA children has also been focused on ghrelin levels in SGA children, a peptide modulating lipid and carbohydrate metabolism and stimulating $\mathrm{GH}$ secretion. It was found that ghrelin concentration is higher in SGA than in AGA newborns, and particularly in SGA children presenting with normal pre-pubertal height, speculating that this may be the adaptive mechanism to stimulate IGF1 production and achieve normal growth in SGA children. Higher ghrelin levels may also increase the risk of long-term morbidity, related to insulin resistance (IR), diabetes mellitus type 2 (DM2) and cardiovascular diseases in adulthood, typical of SGA children and young adults [38]. We recommend, in children born SGA, measurements of weight, length and head circumference every 3 months during the first year and every 6 months during the second year of life. A child who does not show a catch-up growth during the first 6 months of age should receive careful auxological and endocrine monitoring and assessment of GH-IGF1 axis may be required if growth velocity is persistently reduced.

\section{Metabolic characteristics in SGA}

As mentioned above, other than the risk of adult short stature, being born SGA is linked to higher risk of developing Mets (IR, hypertension, and dyslipidemia), and consequently of cardiovascular diseases. IR may be present as early as 1 year of age, and in pre-pubertal age, and it is evident in those with rapid weight gain, probably due to the lower insulin-mediated glucose uptake and insulin sensitivity [39]. The proposed hypothesis to explain the association between LBW and metabolic risk later in life is linked to Barker's 'fetal origin hypothesis:' fetal adaptation to malnutrition in utero may lead to permanent genetic changes resulting in adult morbidities, as the insulin resistance and the reprogramming of the pituitaryadrenal axis results in exposure to higher doses of glucocorticoids $[40,41]$. LBW also seems to be associated with the development of hypertension and cardiovascular diseases in adulthood. A recent case-control study compared AGA newborns and children diagnosed in utero as SGA after 34 weeks of gestation and subdivided into SGA and IUGR categories, according to the absence or presence of weight centile less than 3 or abnormal cerebroplacental Doppler, respectively. Postnatal cardiovascular outcome was evaluated at 3-6 years of age by echocardiography, blood pressure, and carotid ultrasound finding that both SGA and IUGR presented more globular hearts during childhood, reduced longitudinal motion, and impaired relaxation with an increase in radial function. Both groups showed increased blood pressure and carotid intima-media thickness. There was a linear tendency to worse cardiovascular results in IUGR compared with SGA [42]. In general, during childhood, the prevalence of cardiovascular risk factors is low and the routine evaluation of metabolic parameters is not justified for all the SGA born children. Instead, it should be managed following the pediatric guidelines for general population, emphasizing risk increase in adulthood. Regarding body composition, SGA infants usually show low lean body mass and increased central adiposity. Rapid weight gain during childhood is associated with an increased incidence of obesity in adulthood $[43,44]$. For preterm infants, body composition at term equivalent age is different than that of infants born at term. In fact, preterm infants have less lean tissue but similar fat mass [45]. This seems to change during childhood and children born prematurely and full term seem to have similar body composition at preschool age, and the previous differences in terms of body composition at term age were not found at pre-school age in a cohort of 71 children: 21 preterm and 50 term infants [46].

\section{Puberty in SGA}

Although puberty begins at normal age, preterm children usually present earlier onset of pubertal development, faster progression of puberty and earlier menarche compared to fullterm and AGA children. Age of menarche seems also to be related to lower GA. Brandt et al. described a significant difference in age at menarche among preterm SGA girls without catch-up growth and girls born full term and with preterm girls born SGA with postnatal catch-up growth. Height velocity peak is achieved at an earlier pubertal stage and is maintained for a shorter period, increasing the risk of short adult stature. Small bone age delay at the onset of puberty and more rapid bone maturation during puberty has been reported, similar to SGA children, leading to an early fusion of the growth plates and reduced adult height $[47,48]$. The majority of studies investigating the onset of puberty in children born at term SGA and AGA established that, even though puberty begins at an appropriate time (based on chronological age and actual height) in SGA children, its onset happens earlier than in AGA 
children. SGA term children usually present precocious adrenarche, possibly due to some of the major metabolic peculiarities of SGA children with high weight gain in childhood, such as increased central adiposity, decreased insulin sensitivity, and increased IGF-1 levels, that may stimulate androgen secretion. Although there is no complete agreement, several studies show that menarche age in full term SGA female is significantly lower than in AGA girls, however in the normal age range. Regarding bone age and growth spurt, data are similar to the preterm SGA ones, but more long-term followup studies are needed to better understand the biological mechanisms of pubertal development both in preterm and full-term SGA children [49].

\section{Brain development in SGA}

Most of the studies correlating birth weight and neurological outcomes are observational studies with many confounding factors. Nevertheless, several associations between LBW and poor outcomes are known in both in preterm and term infants. Besides the risk of increased mortality and poor outcomes during the first weeks after birth, SGA preterm infants present an additional risk of impaired neurological development in childhood and adulthood. Morsing et al. described the effects of IUGR due to absent or reversed end-diastolic blood flow in the umbilical artery, in very preterm infants (age range 24-29 weeks of gestation) born SGA compared to those born AGA on cognitive outcome at 5-8 years of age. Children born very preterm after IUGR had an increased risk for cognitive impairment at early school age compared with children delivered very preterm for other reasons. Differences in cognitive outcome were restricted to boys who may have been especially vulnerable to the influence of IUGR and very preterm birth [50]. De Jesus et al. also showed, in a large cohort of newborn infants born at $<27$ weeks of gestational age, that compared with non-SGA infants, SGA had higher mortality and were more likely to have postnatal growth failure, prolonged mechanical ventilation, and postnatal steroid use. SGA status was also associated with increased risk of death or neurodevelopmental impairment, presenting with a higher prevalence of cerebral palsy, blindness, cognitive delay, and short stature compared to AGA peers [51]. Growth velocity during hospitalization in NICU also seems also to be associated with the development of cerebral palsy and lower scores at the Bayley Scale at 18-22 months of corrected age [52] and to scholastic performances at eight years of age [53]. Moreover, being born as premature SGA seems to impair cognitive functions, in the areas of mathematics and logic areas, comprehension and attention, and to predispose to hyperactivity disorders, especially when the growth restriction is linked to placental flow alterations [54]. Full-term SGA infants, often unrecognized as 'at risk' population because of birth weight $>2500 \mathrm{gr}$ although lower than the $3 \mathrm{rd}$ centile for gestational age and sex, may also develop long-term neurodevelopmental problems. Several authors have found an increased incidence of minor neurological dysfunction consisting in clumsiness and motor incoordination, hyperactivity, deficit of attention, speech and phonation problems. Considering the high number of full-term SGA infants, neurological consequences may represent a public health concern. A recent nationwide Japanese study examined the impact of SGA birth on behavioral development in a large SGA population, focusing on full-term births. Motor and language development were assessed by questionnaires at 2.5 years of age, and behavioral problems relevant to social development or attention were assessed at 5.5 years of age. SGA status had negative impacts on neurodevelopmental outcomes in full-term children at both ages, consistent with previous studies [55]. Recently, the role of catch-up growth on influencing neurological development was also proposed. Authors divided their 194 SGA population in symmetric and asymmetric groups, showing that the latter had better catch-up growth with larger head circumference. No difference in neurodevelopmental screening was observed between SGA groups, but infants without any catch-up growth were at higher risk of delayed outcome [56]. The assessment of general movements (GMs) during the first 20 weeks of age is a known method for early detection of brain dysfunction and should also be considered also in lowrisk population, as SGA children [57]. GMs in 31 infants with asymmetric intrauterine growth retardation and their appropriate AGA controls were examined and correlated with neurodevelopmental outcome at 2 years. The incidence of normal GMs was lower in the intrauterine growth retarded infants than in the controls, and significant correlations were found between GM quality and neurodevelopmental scores in the intrauterine growth retarded group [58]. In a longitudinal study on the qualitative aspects of GMs of intrauterine growth retarded preterm infants (birth weight below 5th percentile), Bos et al. showed a strong correlation between qualitative assessment of GMs and outcome at 2 years of age, which might help to identify infants at increased risk of neurodevelopmental abnormalities [59]. How much an intrauterine hostile environment influences the neurodevelopment of a preterm baby is still unclear. More studies are needed (with the cooperation of gynecologists, neonatologists and pediatricians) to detect the link between impaired placental functionality, preterm birth, IUGR, and neurocognitive impairment. The GMs assessment, considered a possible precocious marker of neurological wellbeing, may help physicians in detecting who is at higher risk of neurodevelopment impairment.

\section{Conclusion}

In addition to known SGA peculiarities during the first days of life, such as hypoglycemia, thrombocytopenia, increased perinatal morbidity and mortality, full-term and preterm SGA may also contribute to several health consequences, more severe when catch up growth is not achieved. The increasing worldwide percentage of SGA newborns raises a public health concern, which needs prevention measures. All children born SGA should be placed in a multidisciplinary follow up including neonatologists, pediatricians, nutritionists, and endocrinologists, in order to monitor and improve the neurodevelopment and long-term outcomes. Table 3 summarizes the main indications for the SGA clinical follow up. 
Table 3. Auxological, metabolic and neurodevelopmental follow up in SGA children.

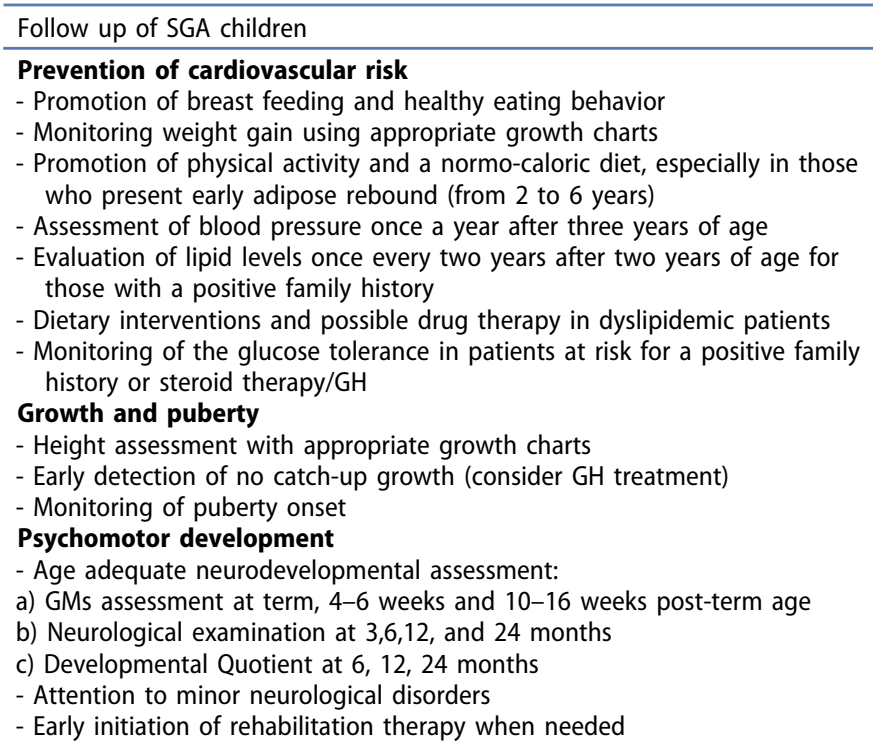

\section{Expert commentary}

Being born SGA, both at term and preterm, is one of the new challenges of the contemporary pediatrician. In fact, this category of children has been shown to be at higher risk of developing cardiovascular and metabolic diseases. The socalled 'noncommunicable diseases' (cardiovascular diseases, diabetes, allergies, osteoporosis, some cancers, etc.) have now become endemic in the western world and trying to improve the diagnostic-therapeutic approach to the babies who are 'at risk' or already have developed the disease is probably not the right key. But why does IUGR influences the newborn's future? During intrauterine life, maternal nutrition and placental integrity play a vital role in the so-called 'metabolic programming:' the different stimuli may cause permanent changes that persist throughout the course of life with long-term implications for the subsequent development of metabolic and neurodevelopmental diseases. These responses are called 'predictive adaptive responses' (PARs) and modulate, through different mechanisms including epigenetic processes, the typical developmental plasticity. Among the PARs we find metabolic control, distribution of adipose tissue, types of muscle fibers, number of cardiomyocytes and nephrons, appetite regulation, responses to stress, timing of pubertal development, and development of central nervous system. Together, they constitute an integrated system that leads the ways in which the adult offspring responds to its environment.

Although PARs should confer adaptive advantages, developmental changes made on the basis of early-life predictions can turn out to be inappropriate: this is already detectable during the first months of life and in early childhood, influencing several clinical aspects of SGA children.

\section{Five-year view}

Advances in prenatal and perinatal medicine will be accompanied by important gains in the rate of survival of infants born preterm and/or IUGR. So far, one in ten newborns in the world are preterm while SGA newborn are about $14 \%$ of the overall births. Physicians involved in the clinical care of this category of children, from neonatologist to pediatric endocrinologists, will need to improve their knowledge to recognize and care for these babies properly.

\section{Key issues}

- Being born with low birth weight (LBW) $(<2500 \mathrm{~g})$ is a condition whose prevalence rises a public health problem, contributing to a variety of short and long-term adverse effects.

- SGA newborn presents weight and/or length below -2 SD or 3rd percentile, and may be classified in two different categories: symmetrical (proportional; 30\%), and asymmetrical (70\%).

- The use of appropriate anthropometric charts is mandatory although modalities of growth change according to intraand extra-uterine growth.

- Most children born SGA recovers percentiles of weight and height after discharge, although they reach a final adult height that is about 1 Standard Deviation lower than the Target Height. About $10-15 \%$ of those born SGA will continue to present short stature (height $\leq-2 \mathrm{SD}$ ) during childhood and adult life and may be eligible for growth hormone treatment.

- Being born SGA is related to higher risk of developing Metabolic Syndrome (Insulin resistance, hypertension, and dyslipidemia), and consequently of cardiovascular diseases in young age.

- Preterm children seem to achieve the complete pubertal development earlier than term peers, worsening final height. In SGA born at term, even though puberty begins at an appropriate time, its onset happens earlier than AGA children, usually presenting precocious adrenarche.

- Although further prospective studies are needed, an increased incidence of minor neurological dysfunction seems to exist in SGA children, both born preterm and at term.

\section{Funding}

No funding declared

\section{Declaration of interest}

The authors have no relevant affiliations or financial involvement with any organization or entity with a financial interest in or financial conflict with the subject matter or materials discussed in the manuscript. This includes employment, consultancies, honoraria, stock ownership or options, expert testimony, grants or patents received or pending, or royalties.

\section{References}

Papers of special note have been highlighted as either of interest $(\cdot)$ or of considerable interest $(\bullet)$ to readers.

1. United Nations Children's Fund. 2003. State of the World's Children Report, 2003. Available from: http://www.unicef.org/sowc03/con tents/pdf/SOWC03-eng.pdf. 
2. Lee $A C$, Katz J, Blencowe $H$, et al. National and regional estimates of term and preterm babies born small for gestational age in 138 lowincome and middle-income countries in 2010. Lancet Glob Health. 2013;1:e26-36.

3. Saenger P, Czernichow $P$, Hughes I, et al. Small for gestational age: short stature and beyond. Endocr Rev. 2007;28:219-251.

4. Clayton PE, Cianfarani S, Czernichow P, et al. Management of the child born small for gestational age through to adulthood: a consensus statement of the International Societies of Pediatric Endocrinology and the Growth Hormone Research Society. J Clin Endocrinol Metab. 2007;92(3):804-810.

- Milestone for diagnosis and treatment of children born small for gestational age.

5. Geva R, Eshel R, Leitner Y, et al. Neuropsychological outcome of children with intrauterine growth restriction: a 9-year prospective study. Pediatrics. 2006;118:91-100.

6. Brandt I, Sticker EJ, Lentze MJ. Catch-up growth of head circumference of very low birth weight, small for gestational age preterm infants and mental development to adulthood. J Pediatr. 2003;142:463-468.

7. Frisk V, Amsel R, Whyte HE. The importance of head growth patterns in predicting the cognitive abilities and literacy skills of smallfor-gestational-age children. Dev Neuropsychol. 2002;22:565-593.

8. Lee PA, Chernausek SD, Hokken-Koelega A, et al. International small for gestational age advisory board consensus development conference statement: management of short children born small for gestational age. Pediatrics. 2003;111:1253-1261.

- A masterpiece in SGA management.

9. Maulik D. Fetal growth compromise: definitions, standards, and classification. Clin Obstet Gynecol. 2006;49(2):214-218.

10. Gardosi J. New definition of small for gestational age based on fetal growth potential. Horm Res. 2006;65(Suppl 3):15-18.

11. Albertsson-Wikland K, Karlberg J. Natural growth in children born small for gestational age with and without catch-up growth. Acta Paediatr Suppl. 1994;399:64-70.

12. Karlberg J, Albertsson-Wikland K. Growth in full-term small-forgestational-age infants: from birth to final height. Pediatr Res. 1995;38(5):733-739.

13. Vaag $A$, Jensen $C B$, Poulsen $P$, et al. Metabolic aspects of insulin resistance in individuals born small for gestational age. Horm Res. 2006;65(Suppl 3):137-143.

14. Blencowe $\mathrm{H}$, Cousens S, Oestergaard MZ, et al. National, regional, and worldwide estimates of preterm birth rates in the year 2010 with time trends since 1990 for selected countries: a systematic analysis and implications. Lancet. 2012;379:2162-2172.

15. Blencowe $H$, Cousens $S$, Chou $D$, et al. Born too soon: the global epidemiology of 15 million preterm births. Reprod Health. 2013;10 (Suppl 1):S2.

16. Engle WA. A recommendation for the definition of 'Late preterm' (Near term) and the birth weight-gestational age classification system. Semin Perinatol. 2006;30(1):2-7.

17. Smith LK, Draper ES, Field D. Long-term outcome for the tiniest or most immature babies: survival rates. Semin Fetal Neonatal Med. 2014;19(2):72-77.

18. Clark RH, Thomas P, Peabody J. Extrauterine growth restriction remains a serious problem in prematurely born neonates. Pediatrics. 2003;111:986-990.

19. Sakurai M, Itabashi K, Sato Y, et al. Hibino S, Mizuno K Extrauterine growth restriction in preterm infants of gestational age $<$ or $=32$ weeks. Pediatr Int. 2008;50:70-75.

20. Pampanini V, Boiani A, De Marchis C, et al. Preterm infants with severe extrauterine growth retardation (EUGR) are at high risk of growth impairment during childhood. Eur J Pediatr. 2015;174 (1):33-41.

-. A very interesting focus on EUGR.

21. Boguszewski MC, Cardoso-Demartini AA. MANAGEMENT OF ENDOCRINE DISEASE: growth and growth hormone therapy in short children born preterm. Eur J Endocrinol. 2017;176(3):R111-R122.

.. Mandatory for doctors involved in prematurity.
22. Fenton TR, Kim JH. A systematic review and meta-analysis to revise the Fenton growth chart for preterm infants. BMC Pediatr. 2013;13:59.

- Interesting meta-analysis for growth charts.

23. Pereira-da-Silva L, Virella D. Is intrauterine growth appropriate to monitor postnatal growth of preterm neonates? BMC Pediatrics. 2014;14:14

24. Fenton TR. A new growth chart for preterm babies: babson and Benda's chart updated with recent data and a new format. BMC Pediatr. 2003;3:13.

25. Cheng YKY, Lu J, Leung TY, et al. Prospective Assessment of the INTERGROWTH-21 and WHO estimated fetal weight reference curve. Ultrasound Obstet Gynecol. 2017. DOI:10.1002/uog.17514

26. Stirnemann J, Villar J, Salomon LJ, et al. International estimated fetal weight standards of the INTERGROWTH-21st Project. Ultrasound Obstet Gynecol. 2016. DOI:10.1002/uog.17347.

27. Bertino E, Spada E, Occhi L, et al. Neonatal anthropometric charts: the Italian neonatal study compared with other European studies. Jpgn. 2010;51:353-361.

- Important for Italian Neonatologists and Pediatric Endocrinologists.

28. Hokken-Koelega AC, De Ridder MA, Lemmen RJ, et al. Children born small for gestational age: do they catch up? Pediatr Res. 1995;38:267-271.

29. Luo ZC, Albertsson-Wikland K, Karlberg J. Length and body mass index at birth and target height influences on patterns of postnatal growth in children born small for gestational age. Pediatrics. 1998;102:E72.

30. Westerberg AC, Henriksen $C$, Ellingvag $A$, et al. First year growth among very low birth weight infants. Acta Paed. 2010;99:556-562.

31. Itabashi K, Mishina J, Tada H, et al. Longitudinal follow-up of height up to five years of age in infants born preterm small for gestational age; comparison to full-term 77 small for gestational age infants. Early Hum Dev. 2007;83:327-333.

32. Monset-Couchard M, De Bethmann O. Catch-up growth in 78166 small-for- gestational age premature infants weighing less than $1000 \mathrm{~g}$ at birth. Biol Neonate. 2000;78:161-167.

33. Bracewell MA, Hennessy EM, Wolke D, et al. The EPICure study: growth and blood pressure at 6 years of age following extremely preterm birth. Arch Dis Child Fetal Neonatal Ed. 2008;93 (2):F108-F114.

34. De Waal WJ, Hokken-Koelega AC, Stijnen T, et al. Endogenous and stimulated GH secretion, urinary GH excretion, and plasma IGF-I and IGF-II levels in prepubertal children with short stature after intrauterine growth retardation. The Dutch Working Group on Growth Hormone. Clin Endocrinol (Oxf). 1994;41:621-630.

35. Boguszewski M, Rosberg S, Spontaneous A-WK. 24- hour growth hormone profiles in prepubertal small for gestational age children. J Clin Endocrinol Metab. 1995;80:2599-2606.

36. Albertsson-WIkland K, Boguszewski M, Karlberg J. Children born small for gestational age: postnatal growth and hormonal status. Horm Res. 1998;49(Suppl 2):7-13.

37. Cutfield WS, Regan FA, Jackson WE, et al. The endocrine consequences for very low birth weight premature infants. Growth Horm IGF Res. 2004;14(Suppl A):S130-S135.

38. Stawerska R, Szałapska M, Hilczer $M$, et al. Ghrelin, insulin-like growth factor I and adipocytokines concentrations in born small for gestational age prepubertal children after the catch-up growth. J Pediatr Endocrinol Metab. 2016;29(8):939-945.

39. Crowther NJ, Cameron N, Trusler J, et al. Association between poor glucose tolerance and rapid post natal weight gain in seven-year old children. Diabetologia. 1998;41:1163-1167.

40. Mericq V, Medina P, Kakarieka E, et al. Differences in expression and activity of 11 beta-hydroxysteroid dehydrogenase type 1 and 2 in human placentas of term pregnancies according to birth weight and gender. Eur J Endocrinol. 2009;161:419-425.

41. Seckl JR, Holmes MC. Mechanisms of disease: glucocorticoids, their placental metabolism and fetal 'programming' of adult pathophysiology. Nat Clin Pract Endocrinol Metab. 2007;3:479-488. 
42. Crispi F, Figueras F, Cruz-Lemini M, et al. Cardiovascular programming in children born small for gestational age and relationship with prenatal signs of severity. Am J Obstet Gynecol. 2012;207 (2):121.e1-9.

43. Baird J, Fisher D, Lucas $P$, et al. Being big or growing fast: systematic review of size and growth in infancy and later obesity. BMJ. 2005;331:929.

44. Monteiro PO, Victora CG. Rapid growth in infancy and childhood and obesity in later life: a systematic review. Obes Rev. 2005;6:143-154.

45. Johnson MJ, Wootton SA, Leaf AA, et al. Preterm birth and body composition at term equivalent age: a systematic review and metaanalysis. Pediatrics. 2012;130(3):e640-9.

46. Scheurer JM, Zhang L, Gray HL, et al. Body composition trajectories from infancy to preschool in children born premature versus fullterm. J Pediatr Gastroenterol Nutr. 2017;64(6):e147-e153

47. Wehkalampi K, Hovi $P$, Dunkel L, et al. Advanced pubertal growth spurt in subjects born preterm: the Helsinki study of very low birth weight adults. J Clin Endocrinol Metab. 2011;96(2):525-533.

48. Lazar L, Pollak U, Kalter-Leibovici O, et al. Pubertal course of persistently short children born small for gestational age (SGA) compared with idiopathic short children born appropriate for gestational age (AGA). Eur J Endocrinol. 2003;149(5):425-432.

49. Verkauskiene R, Petraitiene I, Albertsson Wikland K. Puberty in children born small for gestational age. Horm Res. 2013;80:69-77.

50. Morsing E, Asard M, Ley D, et al. Cognitive function after intrauterine growth restriction and very preterm birth. Pediatrics. 2011;127 (4):e874-e882.

51. De Jesus LC, Pappas A, Shankaran S, et al. Outcomes of small for gestational age infants born at $<27$ weeks' gestation. J Pediatr. 2013;163(1):55-60.
52. Ehrenkranz RA, Dusick AM, Vohr BR, et al. Growth in the neonatal intensive care unit influences neurodevelopmental and growth outcomes of extremely low birth weight infants. Pediatrics. 2006;117(4):1253-1261.

53. Saigal S, Stoskopf BL, Streiner DL, et al. Physical growth and current health status of infants who were of extremely low birth weight and controls at adolescence. Pediatrics. 2001;108 (2):407-415.

54. A-K VB, Kollmann M, Rotky-Fast C, et al. Perinatal complications and long-term neurodevelopmental outcome of infants with intrauterine growth restriction. Am J Obstet Gynecol. 2013;208:130.e1-6.

55. Takeuchi A, Yorifuji T, Takahashi K, et al. Neurodevelopment in fullterm small for gestational age infants: a nationwide Japanese population-based study. Brain Dev. 2016;38(6):529-537.

56. Maciejewski E, Hamon I, Fresson J, et al. Growth and neurodevelopment outcome in symmetric versus asymmetric small for gestational age term infants. J Perinatol. 2016;36(8):670-675.

57. Einspieler C, Prechtl HFR, Bos AF, et al. Prechtl's method on the qualitative assessment of general movements in preterm, term and young infants. Clin Dev Med. 2004;167:1-91.

-. A milestone for understanding general movements.

58. Zuk L, Harel S, Leitner Y, et al. Neonatal general movements: an early predictor for neurodevelopmental outcome in infants with intrauterine growth retardation. J Child Neurol. 2004;19 (1):14-18.

59. Bos AF, Van Loon AJ, Hadders-Algra M, et al. Spontaneous motility in preterm, small-for-gestational age infants. II. Qualitative aspects. Early Hum Dev. 1997;50(1):131-147.

- New horizons in GMs clinical application. 